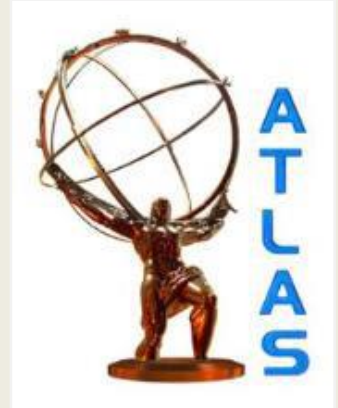

\title{
Measurement of the jet production properties at the LHC with the ATLAS Detector
}

S. Tokar, Comenius University, Bratislava

On behalf of the ATLAS collaboration

EDS Blois 2015: The $16^{\text {th }}$ conference on Elastic and Diffractive scattering Borgo, Corsica, France 2015 


\section{Outline of the talk}

$\square$ Jet physics motivation

$\square$ ATLAS detector

$\square$ Dijet cross section in pp collisions at $7 \mathrm{TeV}$

$\square$ Limits on contact interaction

$\square$ Dijet flavour decomposition

$\square$ Inclusive jet cross section in pp collisions at $7 \mathrm{TeV}$

$\square$ Three-jet production in pp collision at $7 \mathrm{TeV}$

$\square$ Jet shapes in ttbar events

$\square$ Conclusions 


\section{Jet physics motivation}

Test of the quantum chromodynamics ( $Q C D)$ : jets are the result of fragmentation of partons produced in a scattering process.

In high-energy particle collisions - two main phases:

$\checkmark$ Perturbative phase: partons with high-transverse momentum $\left(p_{\mathrm{T}}\right)$ are produced in a hard-scattering process at a scale $Q$.

$\checkmark$ Non-perturbative phase: partons convert in hadrons emitting gluons and $q \bar{q}$-pairs - non-perturbative part of jet evolution is an interplay between hadronisation process (HP) and underlying event (UE).

- Hadronisation process: transition from partons to hadrons

- Underlying event: initial-state radiation, multiple-parton interactions and colour-reconnection effects.

- Effects of HP and UE vary strongly with the jet radius parameter and are most pronounced at low $p_{T}$.

All these aspects of high energy collisions can be probed in the jet physics! 


\section{Atlas experiment}

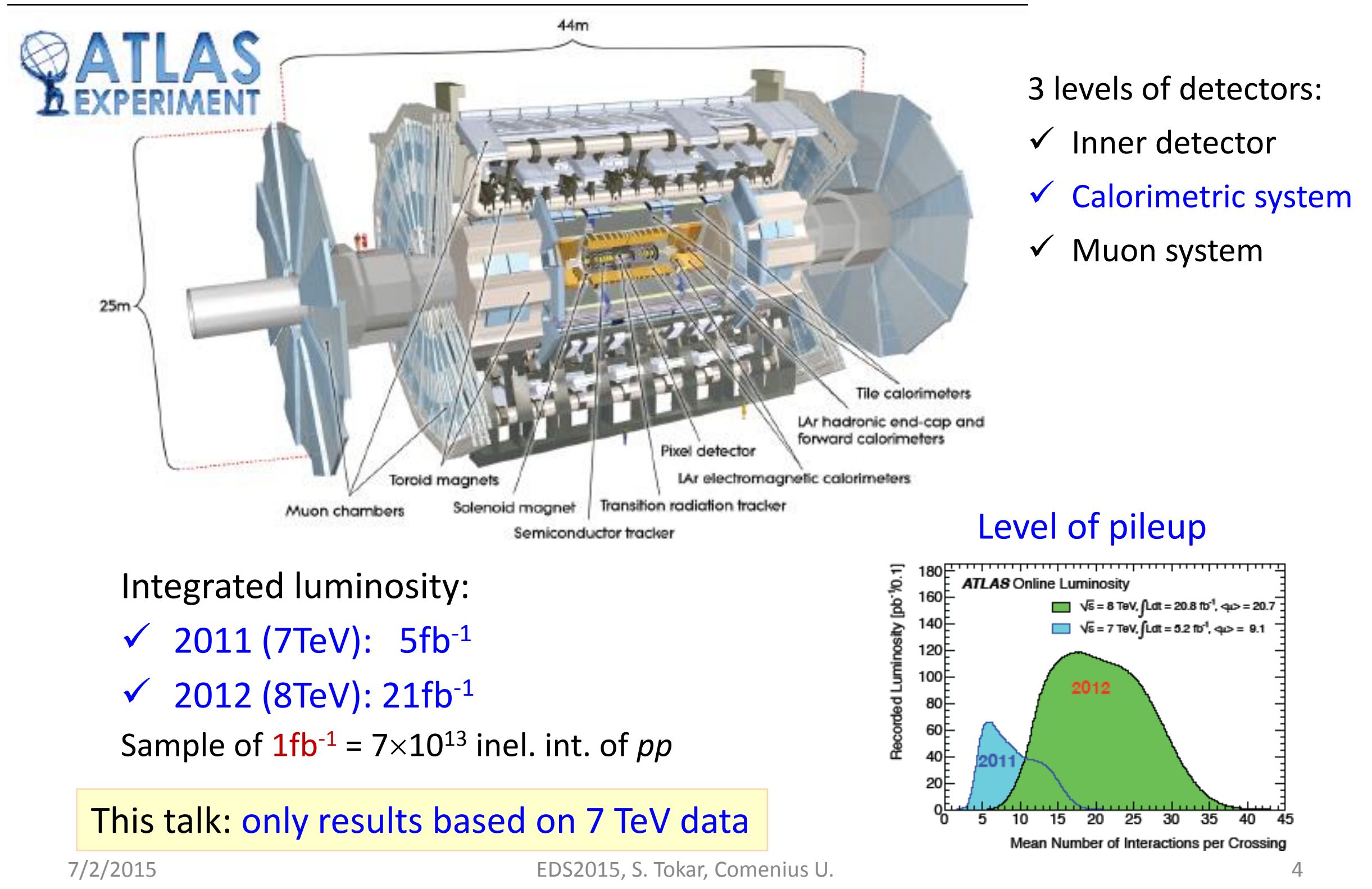




\section{Dijet cross-sections in $p p$ at $7 \mathrm{TeV}$}

$\checkmark$ Jets reconstructed by the anti- $k_{t}$ algorithm JHEP 05(2014)059 jets are clustered using two different values of R: 0.4 and 0.6 .

$\checkmark$ Measured cross-sections are corrected for all experimental effects and are defined at the particle-level final state (unfolding to particle level jets).

$\checkmark$ A set of single-jet triggers with different thresholds used to collect data. Offline: Leading (subleading) jet required: $p_{\mathrm{T}}>100 \mathrm{GeV}$ ( > $50 \mathrm{GeV}$ ).

$\checkmark$ Only events collected during stable beam conditions - at least one primary vertex, reconstructed $\geq 2$ tracks with $\mathrm{p}_{\mathrm{T}}>400 \mathrm{MeV}$.

$\checkmark$ Primary vertex with the highest $\sum{p_{T}}^{2}$ of associated tracks is selected as the hard-scatter vertex.

$\checkmark$ Dominant uncertainties: jet energy calibration - from balance in $\mathrm{Z} / \gamma$-jet , dijet intercalibration, multijet balance and single hadron response. 


\section{Dominant uncertainty: Jet Energy Scale}

EPJC 75(2015)17

$\checkmark$ Jets reconstructed by the anti- $k_{t}$ algorithm - and clustered using $\mathrm{R}=0.4$.

$\checkmark$ Fractional JES uncertainty vs jet $p_{\mathrm{T}}$ for $\eta=0.5$ and $\eta=2.0$.
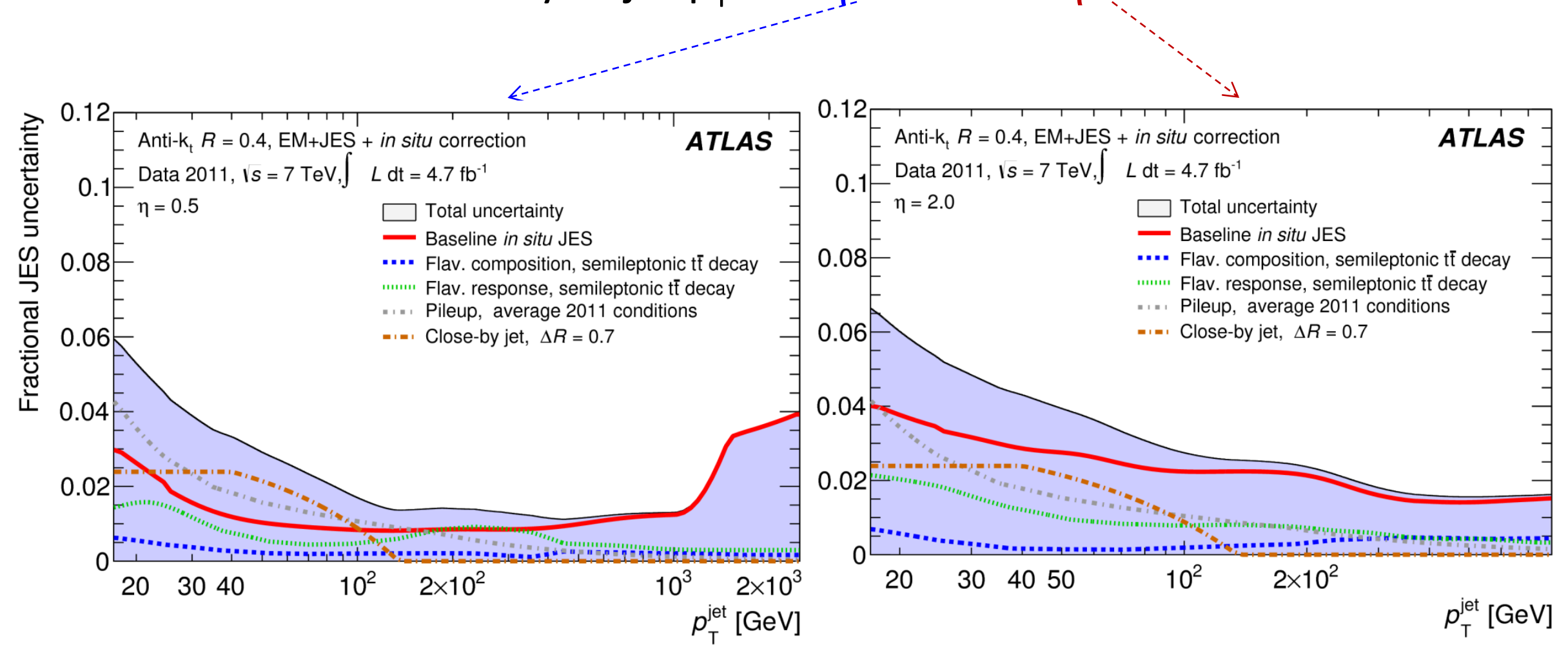

In situ JES uncertainty comes from measurements of $Z+$ jets, $\gamma+$ jets, multijet balance, $\eta$-intercalibration, single hadron. 


\section{Particle level vs parton level X-sections}

Measured X-sec are compared to NLO QCD predictions corrected for $\checkmark$ non-perturbative effects in fragmentation process and in underlying event. $\checkmark$ NLO electroweak effects.

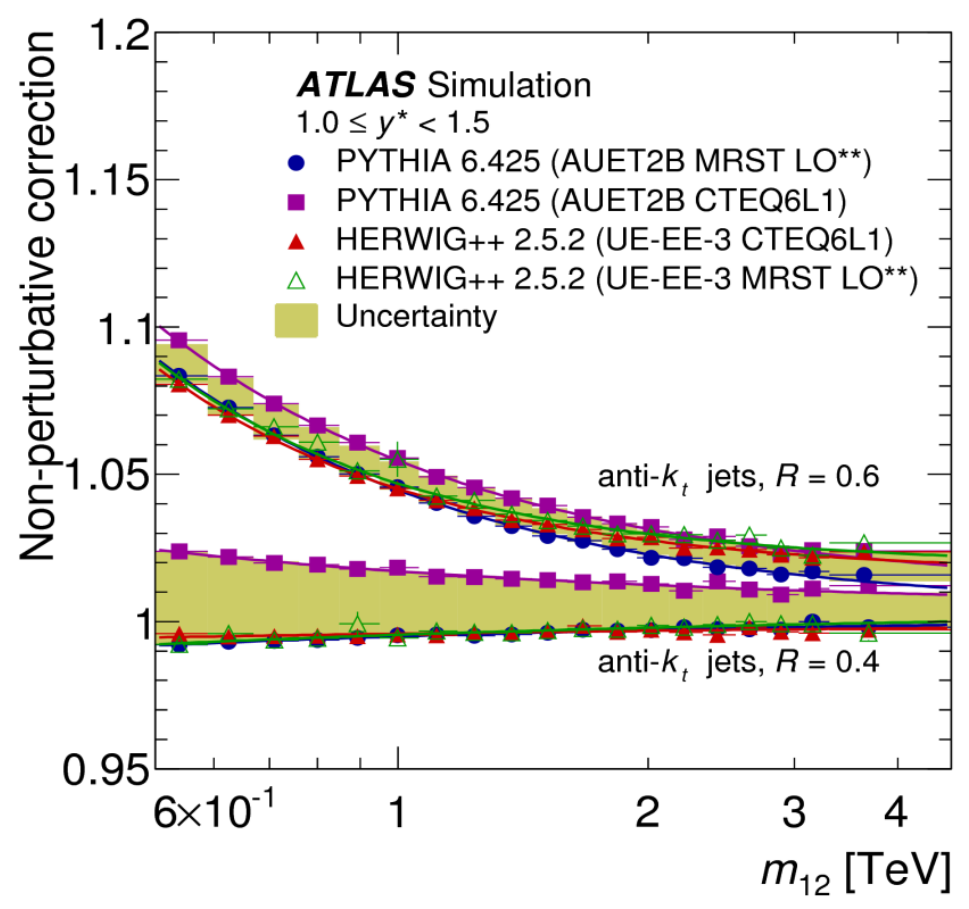

Non-perturbative effects (depending on the jet R): evaluated using leading-log generator (Pythia 6.425...)

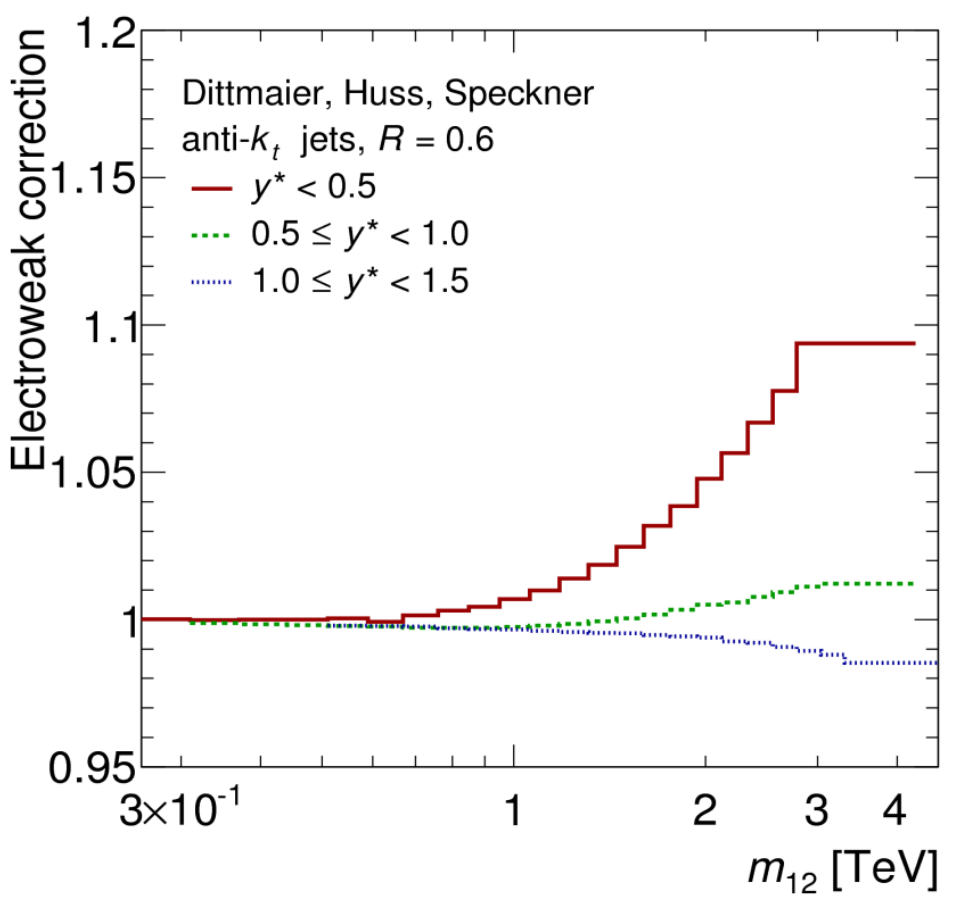

EW corrections for tree-level + weak loop effects are applied to the NLOJet++ and POWHEG predictions 


\section{Dijet cross section}

Measurements of the dijet double-diff. cross-sections vs dijet mass and rapidity separation $\left(y^{*}=\left|y_{1}-y_{2}\right| / 2\right)$ - anti- $k_{t}$ jets with $R=0.4$ and $R=0.6$.

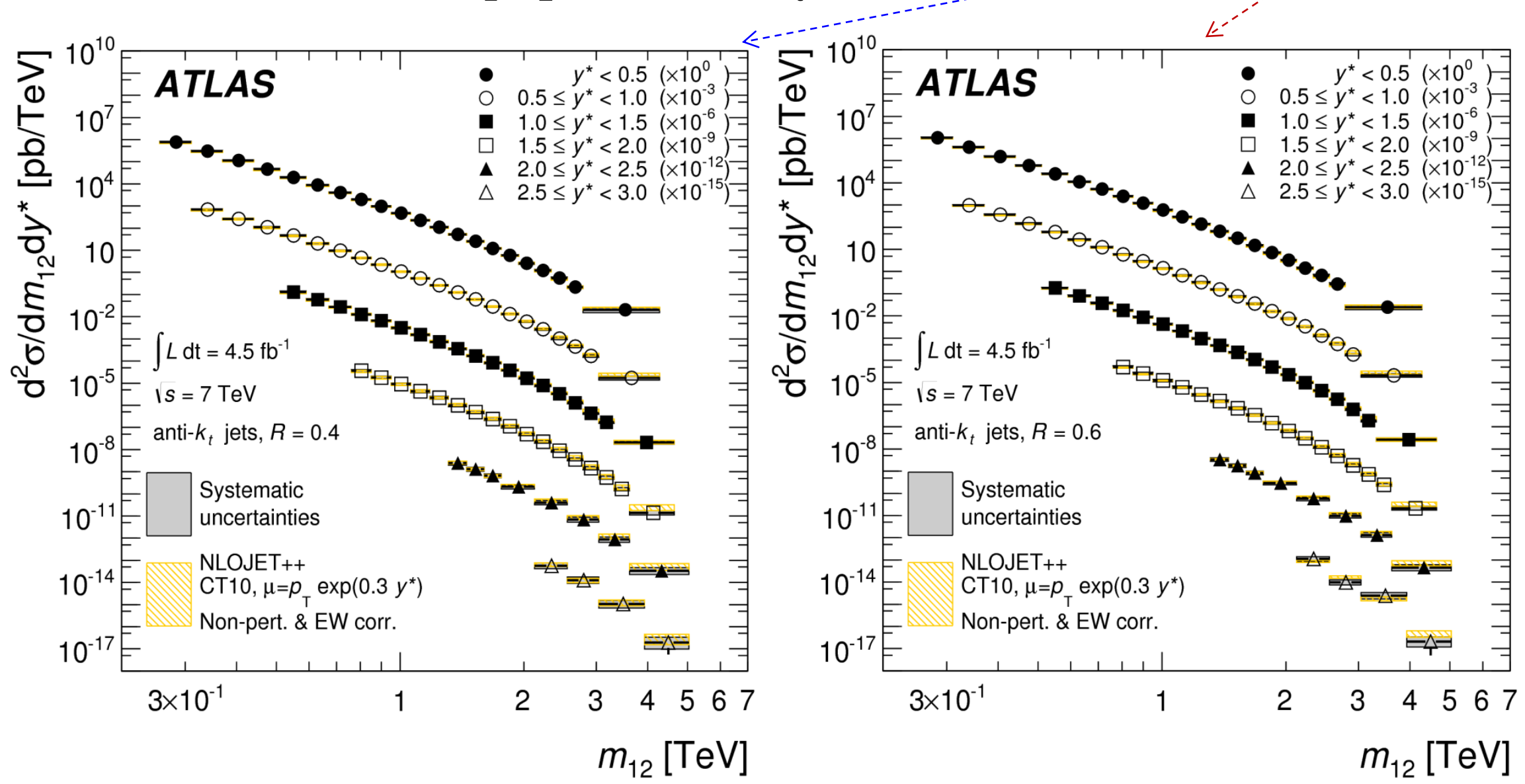

Data are compared to NLOJet++ predictions with various PDFs (CT10), corrected for non-perturbative and EW effects, scale: $\mu=\mu_{\mathrm{R}}=\mu_{\mathrm{F}}=p_{T}{ }^{\max } e^{0.3 y^{*}}$. 


\section{Dijet cross section}

Ratio of NLOJet++ prediction to measurements of dijet double-diff. X-sec vs dijet mass and $\mathrm{y}^{*}$ - PDF sets used: CT10, HERAPDF1.5, and epATLJet13.

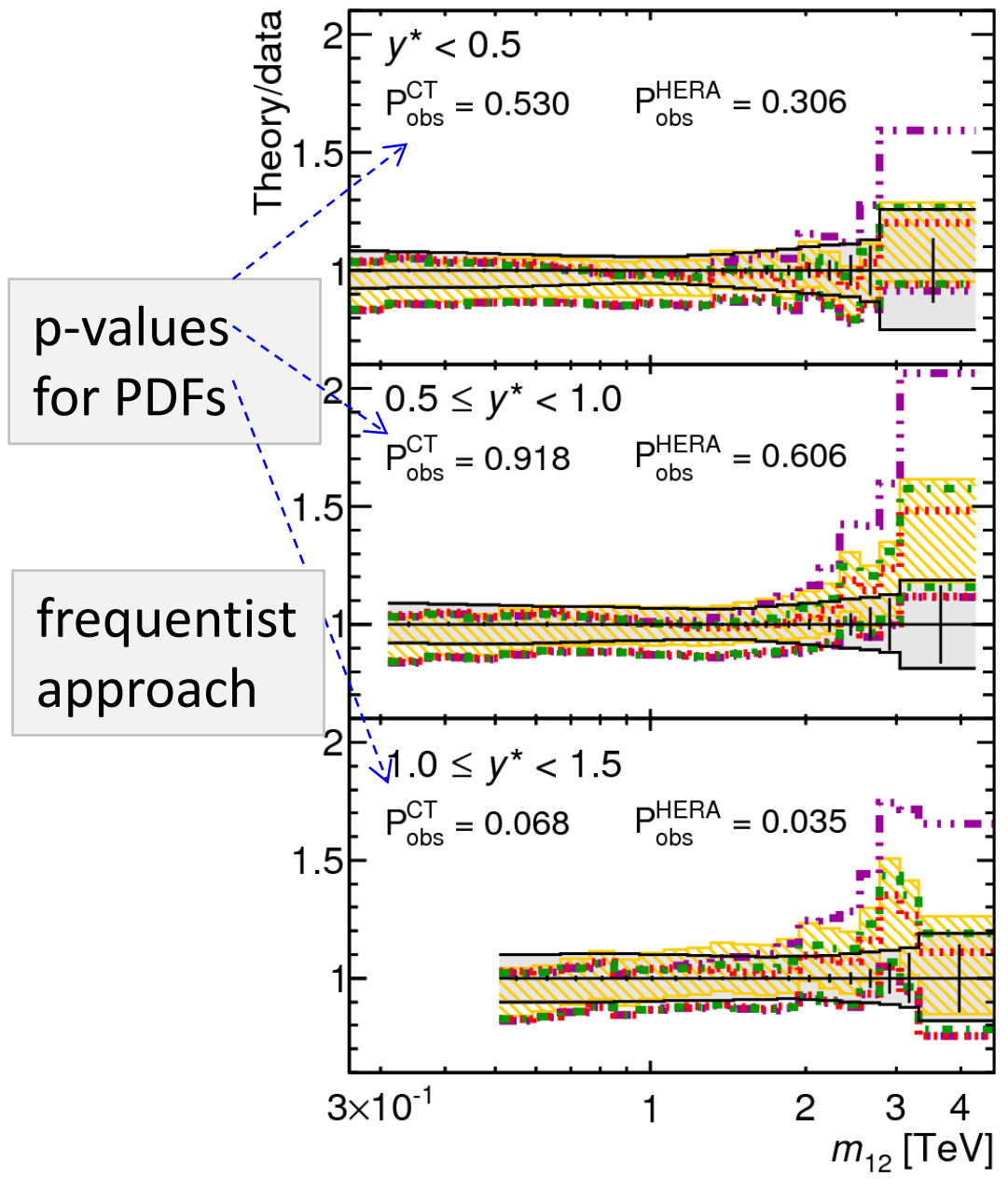

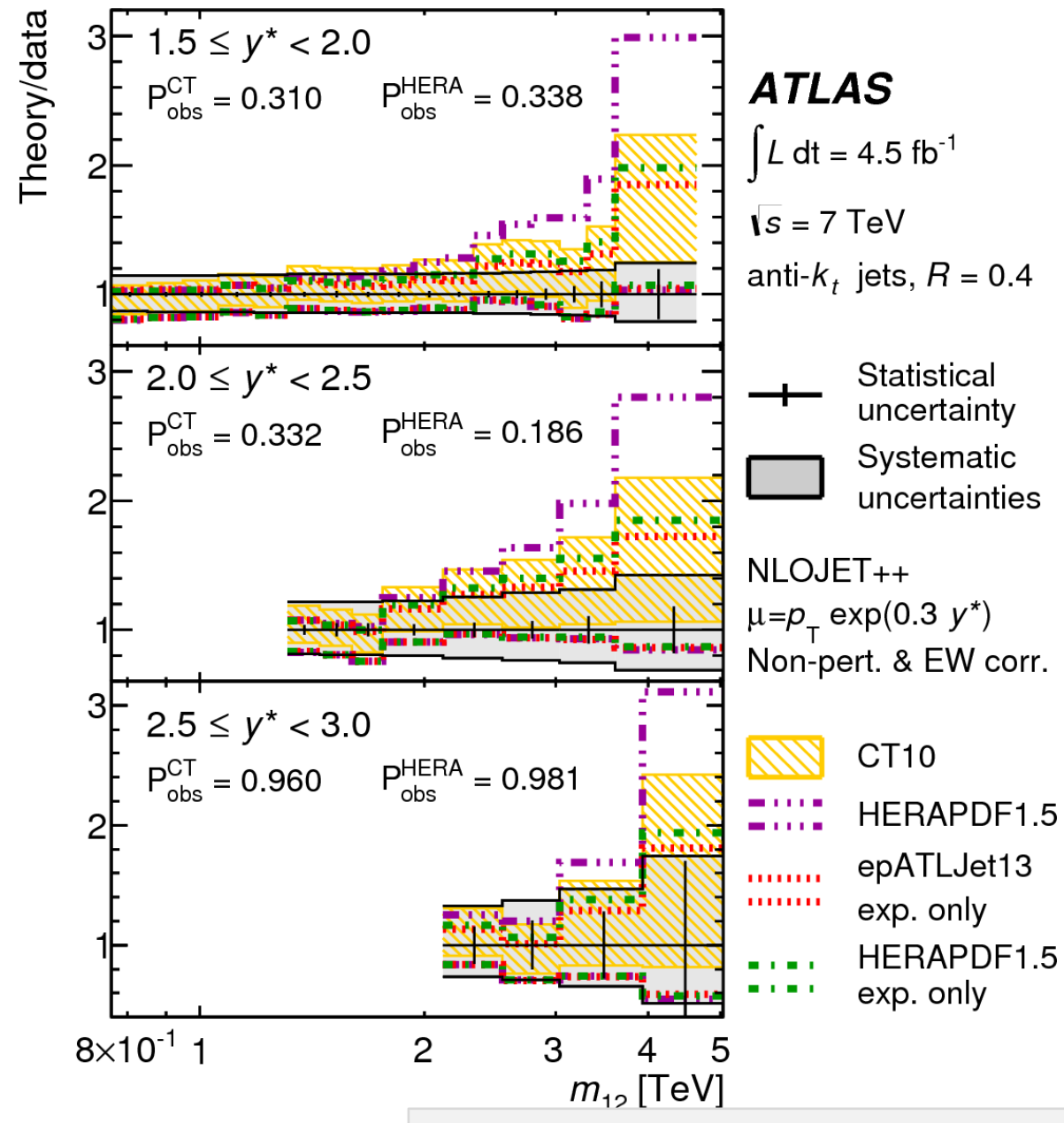

Potential for PDF improvement 


\section{Exclusion of contact interactions}

$\checkmark$ If quarks are composed of more fundamental particles with new strong interactions at a composite scale $\Lambda$ ( $\left.>\mathrm{m}_{\text {quark }}\right) \Rightarrow$ at energies $<<\Lambda$ : contact interaction from the underlying strong dynamics $\rightarrow$ observable effect.

$\checkmark$ Model of QCD + contact interactions (Cls) with left-left coupling and destructive interference between $\mathrm{Cls}$ and $\mathrm{QCD}$ is considered.

$\checkmark$ Measurement is restricted to the high dijet-mass $m_{12}>1.31 \mathrm{TeV}$ in the range $\quad y^{*}<0.5\left(\right.$ Cls: events preferentially produced at smaller $\left.y^{*}\right)$.

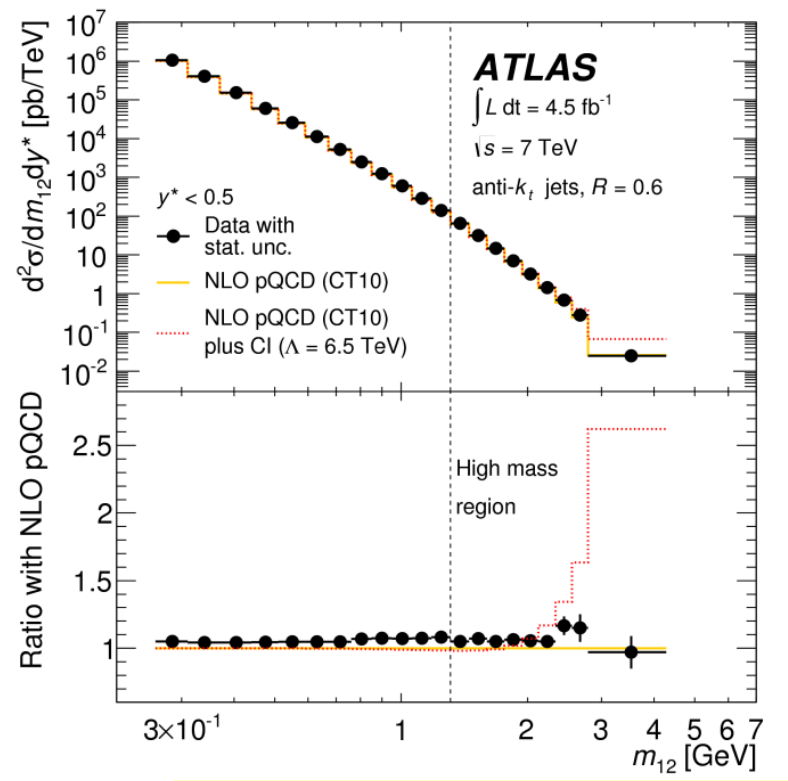

\begin{tabular}{|l|c|c|}
\hline \multicolumn{2}{|l|}{ PDF set } & \multicolumn{2}{|c|}{$\mathrm{R}=0.6$} \\
\hline \multicolumn{1}{|c|}{$\Lambda[\mathrm{TeV}]$} & \multicolumn{1}{|c|}{ Exp } & Obs \\
\hline CT10 & 7.1 & 7.1 \\
\hline HERAPDF1.5 & 7.3 & 7.7 \\
\hline MSTW 2008 & 7.1 & 6.9 \\
\hline NNPDF2.1 & 7.2 & 7.0 \\
\hline
\end{tabular}

Quark size $<3 \cdot 10^{-20} \mathrm{~m}$

Measured cross section vs NLO QCD (yelow line) and NLO QCD + Cls with $\Lambda=6.5 \mathrm{TeV}$ (red line) predictions, CT10 PDF set used. 


\section{Dijet flavour decomposition}

Flavor decomposition measurement: pp collision at $V_{s}=7 \mathrm{TeV}, L_{\mathrm{int}}=39 \mathrm{pb}^{-1}$. Decomposition determined in 6 jet $\mathrm{pT}$ bins

Heavy quarks participating in the hard scattering:

$\checkmark$ Quark pair creation: $g g \rightarrow Q \bar{Q}$ and $q \bar{q} \rightarrow Q \bar{Q}$.

$\checkmark$ Heavy flavour quark excitation: a single heavy quark from the sea of one hadron scatters on a parton from another hadron: $g Q \rightarrow g Q$ and $q Q \rightarrow q Q$.

Additional motivation $\Rightarrow$ sensitivity to proton sea heavy quarks

$\checkmark$ Gluon splitting: $g \rightarrow Q \bar{Q}$.

PYTHIA 6.423:

The relative contributions of the different heavy flavor quark production mechanisms to inclusive $b$-jet production for simulated pp collisions at $7 \mathrm{TeV}$; jets in $|y|<2.1$.

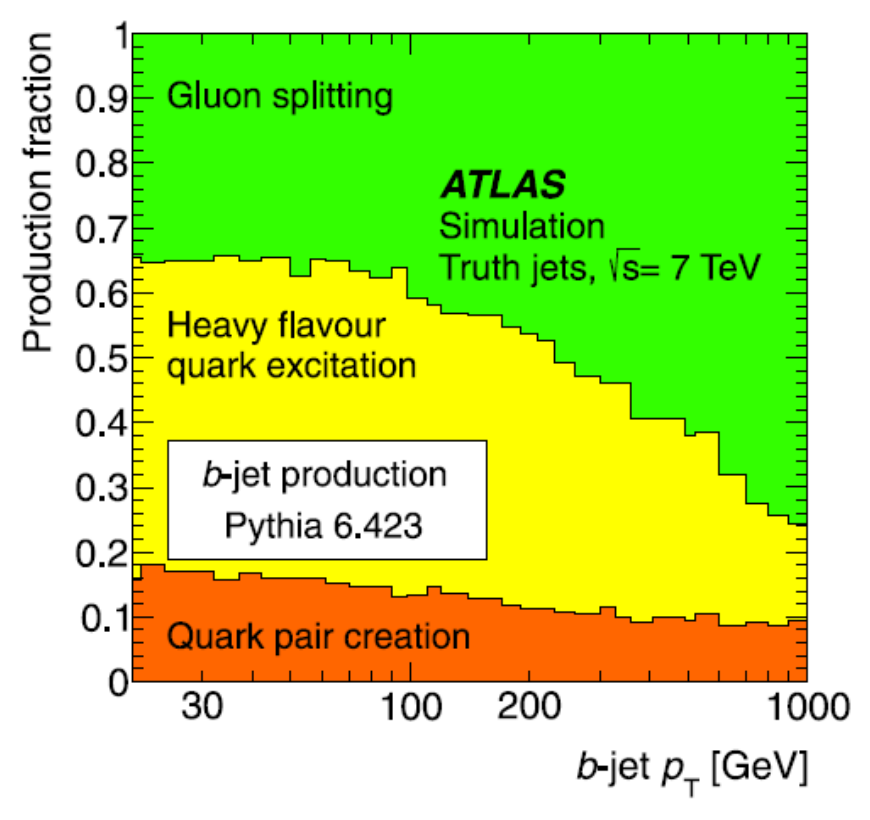




\section{Dijet flavour: predictions and reconstruction}

\section{Classification $\Rightarrow$}

Light quarks (U): up, down and strange, Heavy quarks: charm (C) and bottom (B)

Kinematic properties of the produced partons are mostly flavour independent .

A single secondary vertex is fitted for each jet.

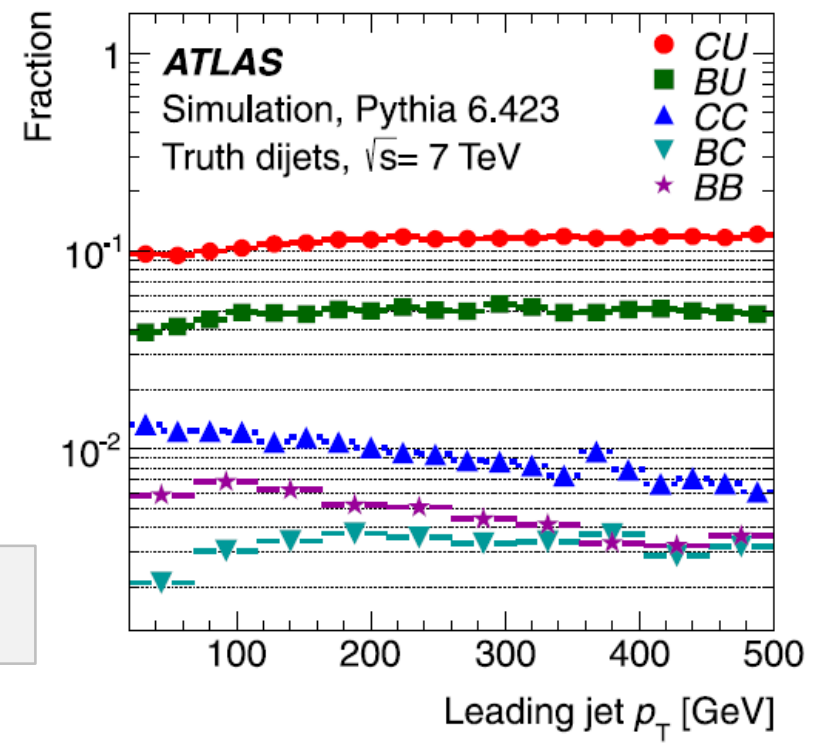

To distinguish jet flavour: $\Pi=\frac{m_{\text {vertex }}-0.4 \mathrm{GeV}}{m_{B}} \cdot \frac{\sum_{\text {vertex }} E_{i}}{\sum_{\text {jet }} E_{i}}$,

$$
B=\frac{\sqrt{m_{B}} \cdot \sum_{\text {vertex }}\left|\vec{p}_{T}\right|}{m_{\text {vertex }} \cdot \sqrt{p_{\text {Tet }}}}
$$

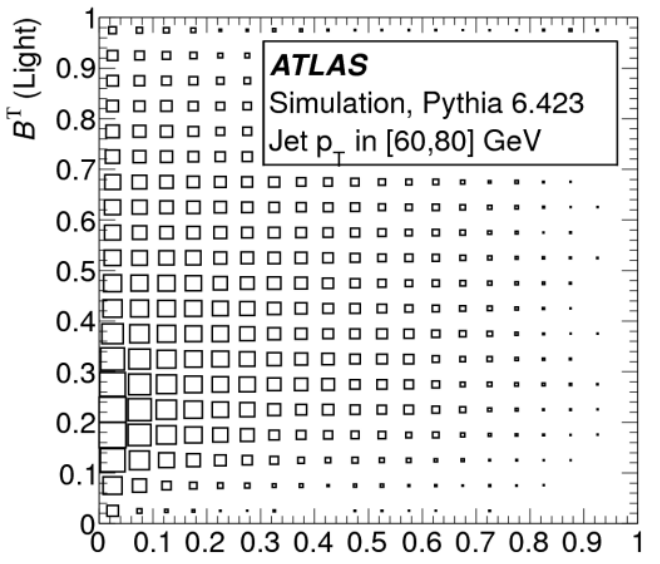

$\Pi^{\mathrm{T}}$ (Light)

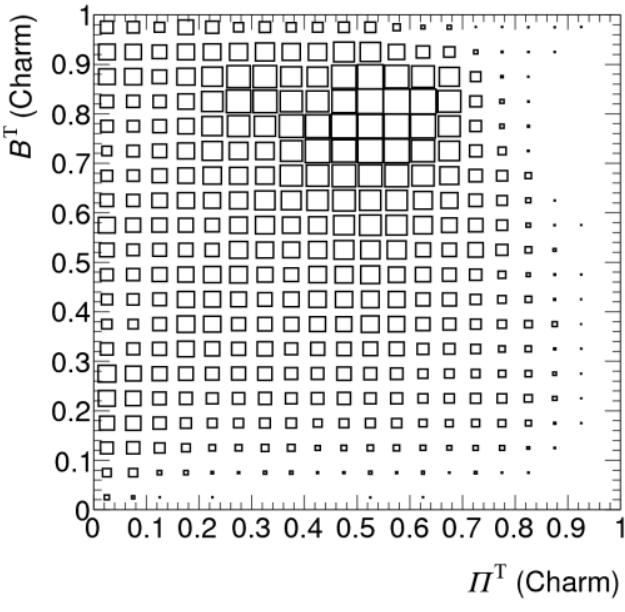

EDS2015, S. Tokar, Comenius U.

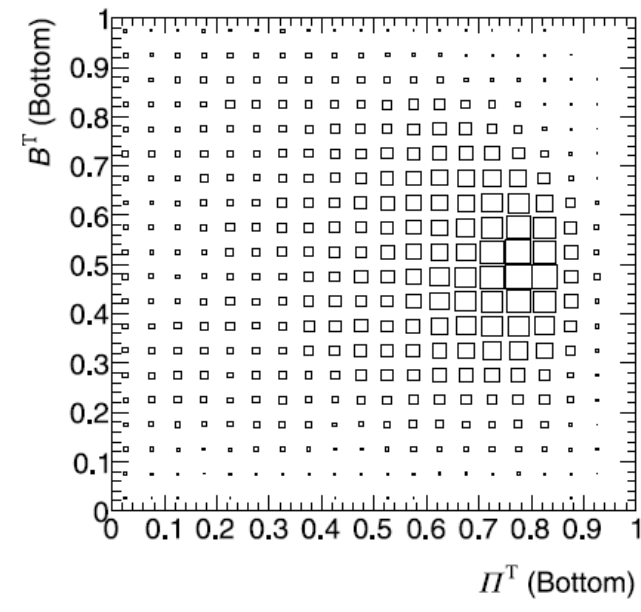




\section{Dijet flavour: results}

\section{Dijet production fraction: Data vs MC (Pythia 6.423, Herwig++ 2.4.2, Powheg+Pythia)}

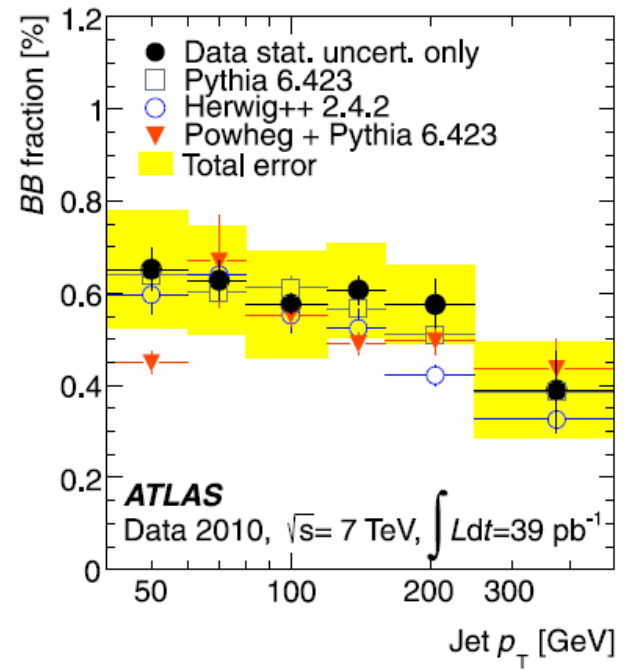

(a)

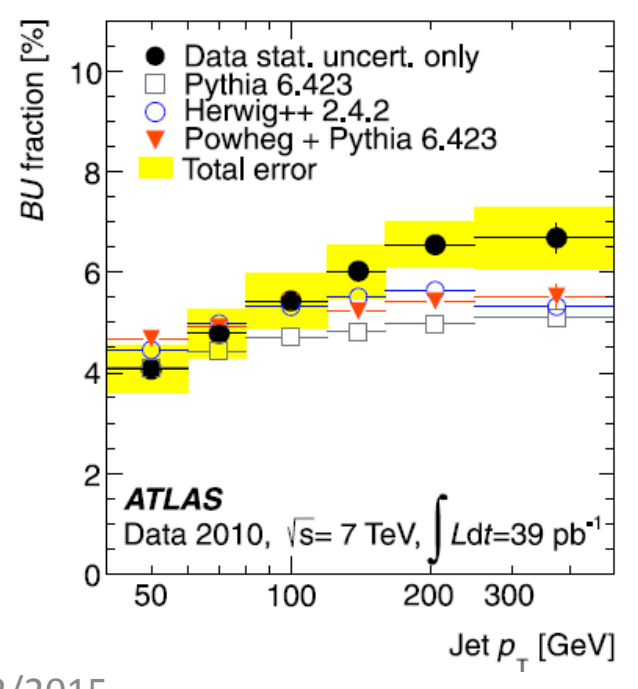

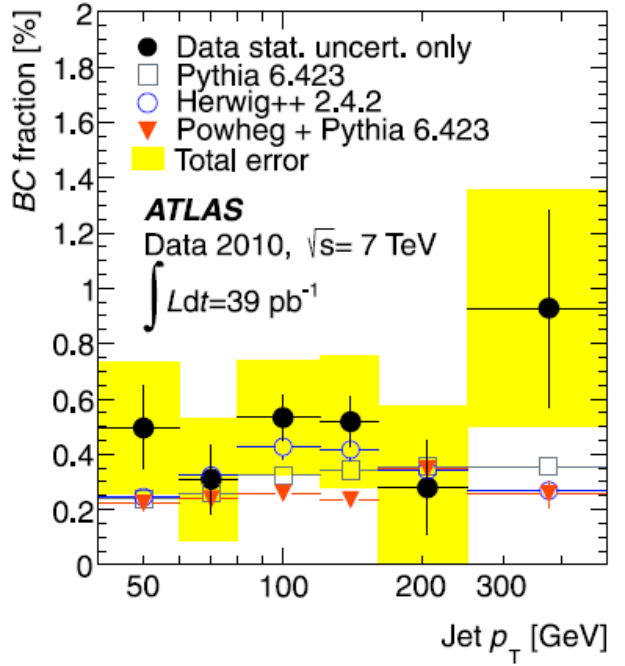

(b)

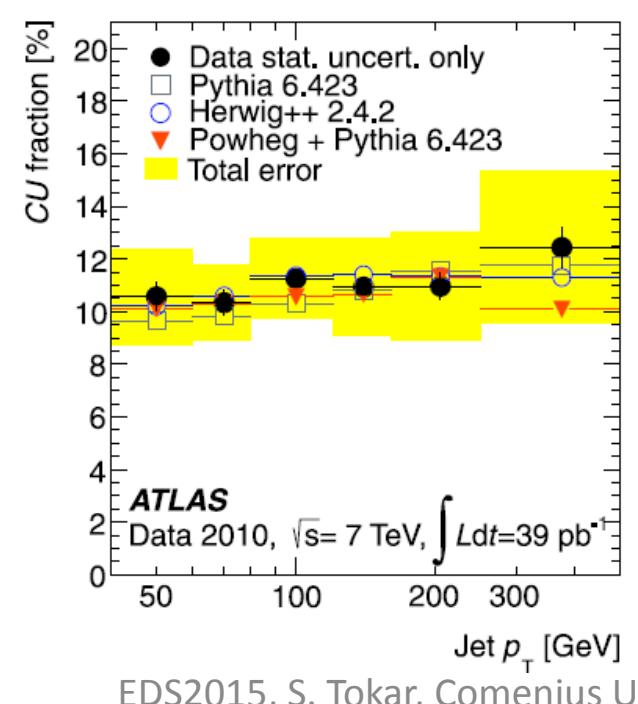

EDS2015, S. Tokar, Comenius U

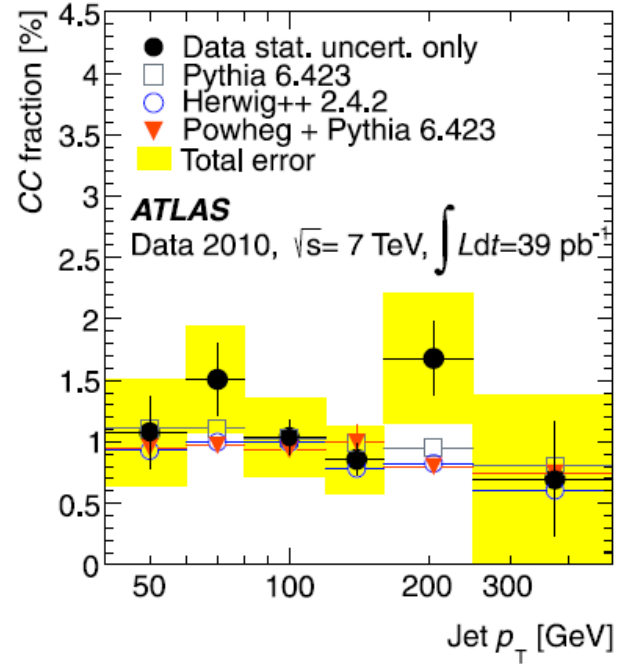

(c)

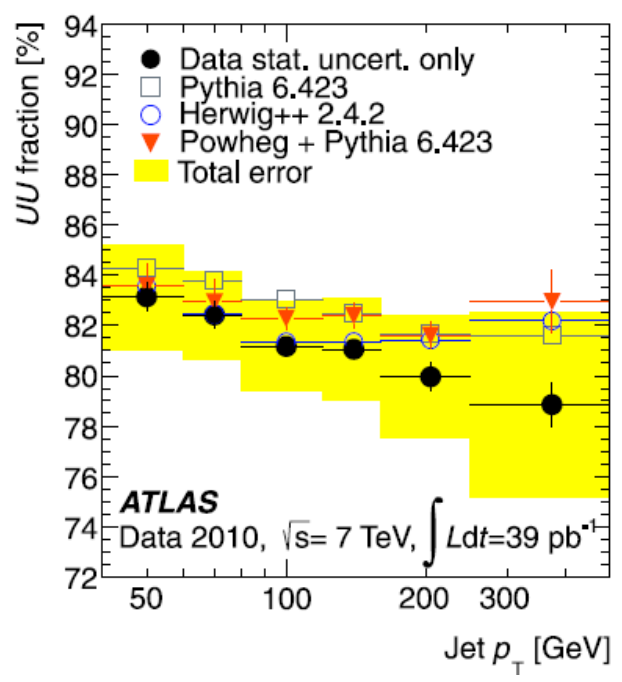




\section{Inclusive jet cross-section in $p p$ at $7 \mathrm{TeV}$}

$\Rightarrow$ Motivation: a test of validity of $\mathrm{pQCD}$ and probing of the parton distribution functions (PDFs) in the proton.

JHEP 02 (2015) 153

$\checkmark$ Uncertainty in the NLO pQCD prediction of incl. Xsec vs jet $p_{T}$.

$\checkmark$ Jet cross section unfolded to particle-level jets to compare them with NLO pQCD: corrections for non-perturbative and EW effects is done.

$\checkmark$ Theoretical predictions: NLO pQCD calculated by NLOJET++ 4.1.2 with several PDFs and different $\mu_{\mathrm{R}}$ and $\mu_{\mathrm{F}}$ - to cover missing high.-o. corrections.

$\checkmark$ Experimental systematic uncertainties: Main sources: JES and jet energy resolution. For $|y|<0.5$ and $p_{T}<1$ TeV less than $10 \%$ -
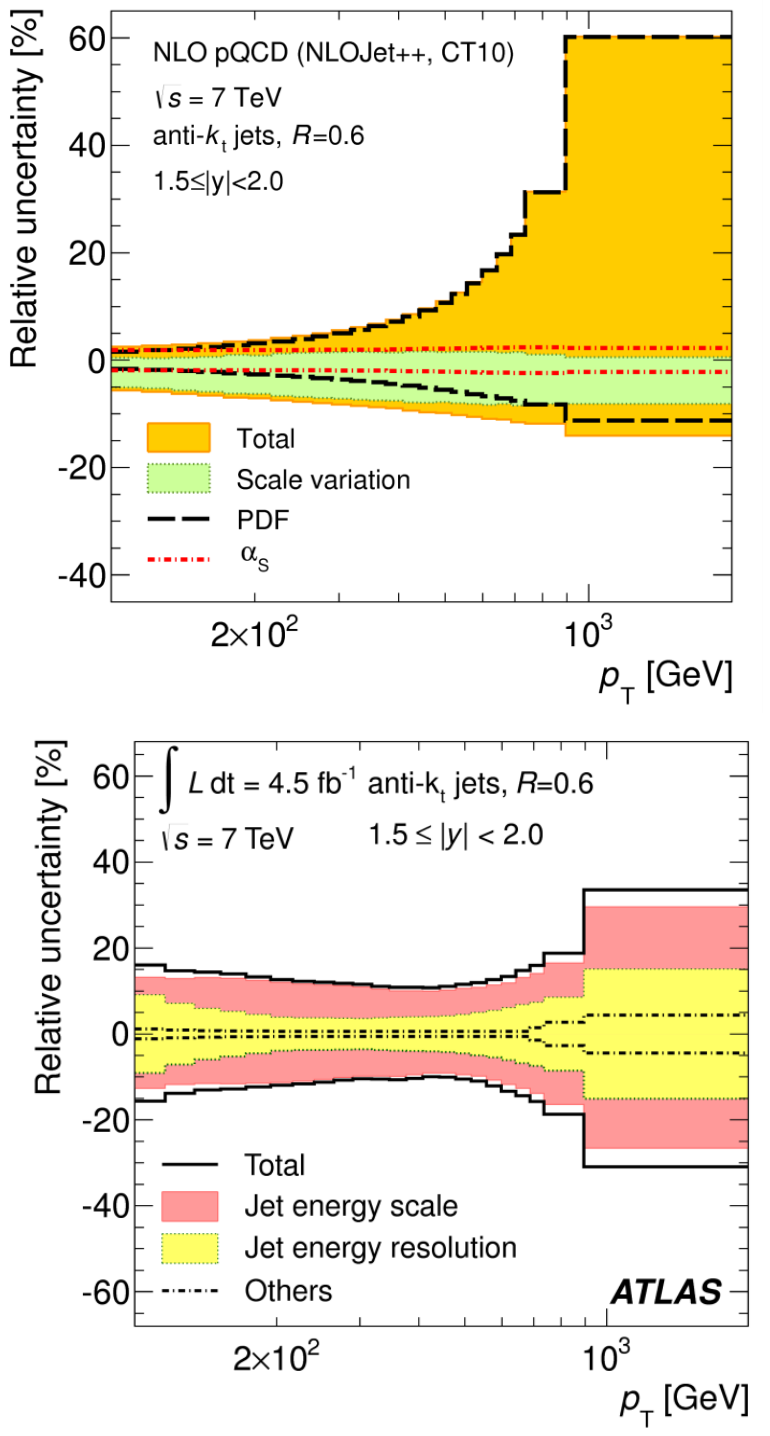


\section{Inclusive jet cross-section in $p p$ at $7 \mathrm{TeV}$}

Double-diff. inclusive jet $X$-sections for jets with $\mathrm{R}=0.4$ vs jet $\mathrm{p}_{\mathrm{T}}$ and rapidities $\Rightarrow$ data vs NLO pQCD prediction corrected for non-perturb. and EW effects
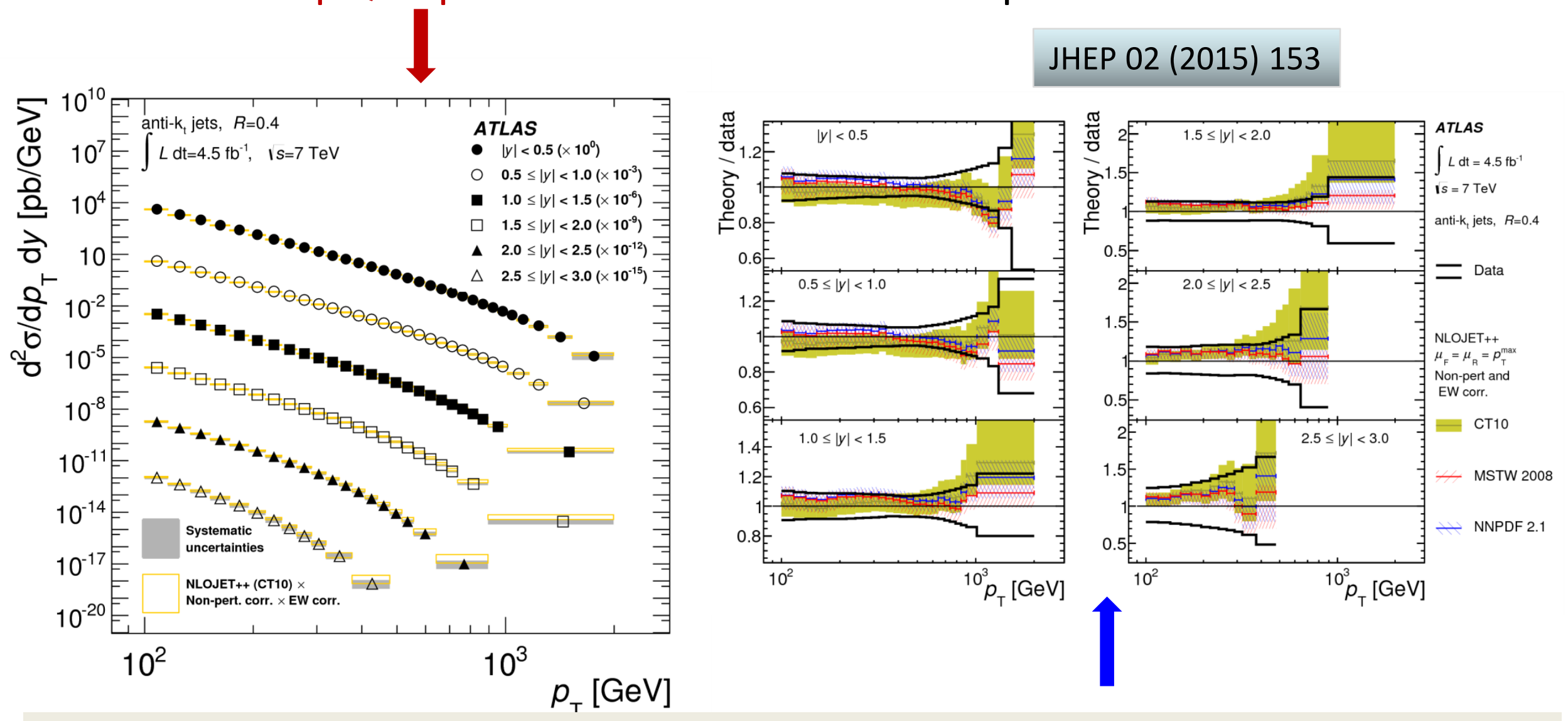

Ratio of NLO pQCD predictions to measured double-diff. inclusive jet $X$-section vs jet $\mathrm{P}_{\mathrm{T}}$ and jet rapidity - different NLO PDF sets used: CT10, MSTW2008 and NNPDF 2.1. 


\section{3-jet production cross-section in $p p$ at $7 \mathrm{TeV}$}

Motivation: study of higher order effects of $p Q C D$

$\square$ 3-level jet trigger used: events with $\geq 3$ jets - leading, subleading and subsubleading jets are required: $\boldsymbol{p}_{\mathrm{T}}>150 \mathrm{GeV}, \boldsymbol{p}_{\mathrm{T}}>100 \mathrm{GeV}$ and $\boldsymbol{p}_{\mathrm{T}}>50 \mathrm{GeV}$.

$\square$ 3-jet x-sections vs $\boldsymbol{m}_{j j j}$ in 5 bins of $\left|Y^{*}\right|\left(=\left|y_{1}-y_{2}\right|+\left|y_{2}-y_{3}\right|+\left|y_{1}-y_{3}\right|\right)<10-$ obtained by unfolding the data distributions to particle level.

Experimental uncertainties: dominant is JES, jet energy and angular resolution $\Rightarrow$ $8-10 \%$ for low $\boldsymbol{m}_{j j j}, 28 \%$ at high $\boldsymbol{m}_{j j j}$ at $\left|Y^{*}\right|<6$.

Theo. predictions and uncertainties: NLO QCD predictions (NLOJET++). Scale: $\mu_{\mathrm{R}}=\mu_{\mathrm{F}}=m_{\mathrm{jjj}}$.

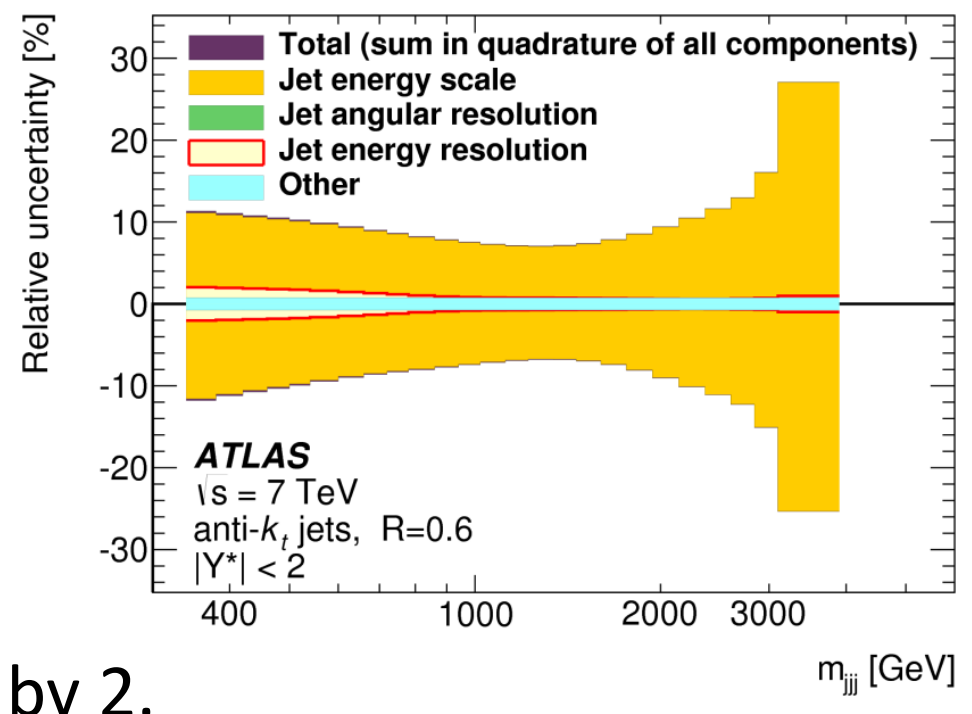
Uncertainty due to missing h.o.: scale up/down by 2 . 


\section{3-jet production cross-section at $p p 7 \mathrm{TeV}$}

3-jet double diff. $x$-sec vs $m_{j j j}$ in 5 bins of $\left|Y^{*}\right|$ for jets with $R=0.6$ compared with NLOJET++ prediction with CT 10 PDF corrected for non-perturb. effects
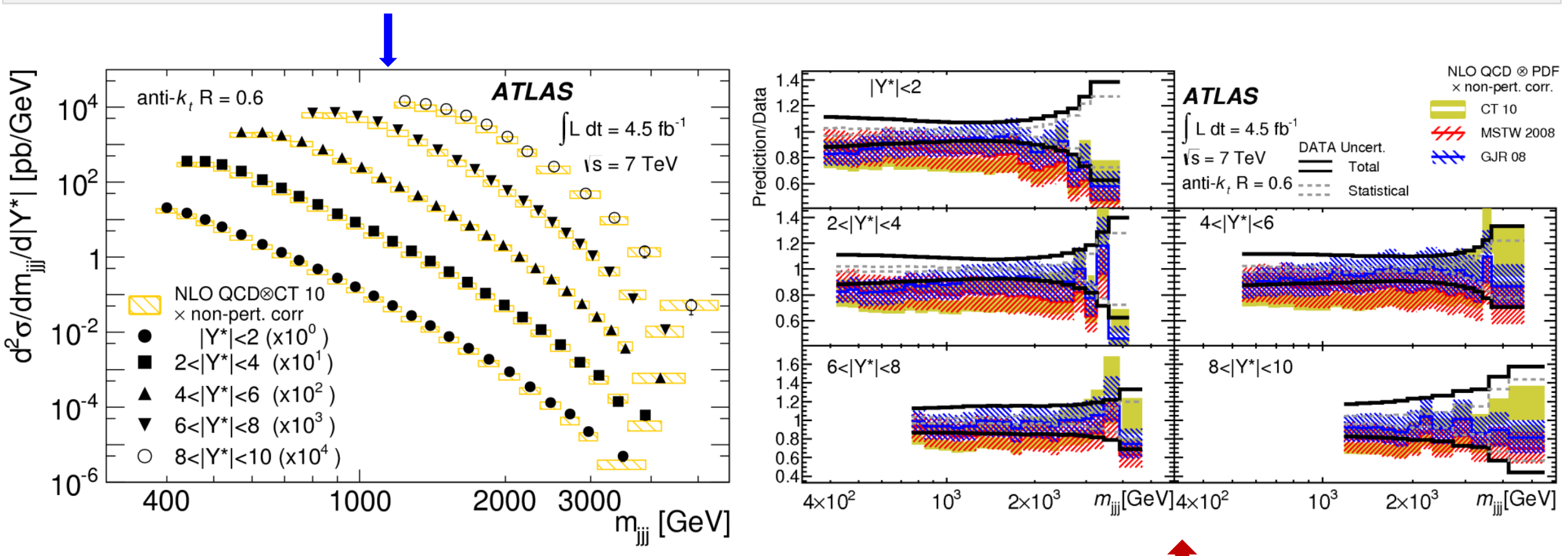

The ratio of NLO QCD predictions, obtained by using NLOJET++ with different PDF sets (CT 10, MSTW 2008, GJR 08) and corrected for non-perturbative effects, to data as a function of $m_{j j j}$ in 5 bins of $\left|Y^{*}\right|$. 


\section{Jet shapes in tt-bar events in $p p$ at $7 \mathrm{TeV}$}

Motivation: in $t \bar{t}$ events jets are observed at large momentum-transfer $\Rightarrow$ a good place for study of jet shapes - to understand perturbative and nonperturbative phase of jet evolution.

Aimed at $t \bar{t}$ final state $b$-jets ( $\rightarrow \mathrm{Wb}$ ), in lepton+jets and di-lepton channels, and light jets from $W^{+} \rightarrow u \bar{d}, c \bar{s}+$ C.C.

Two sample of jets selected:

$\checkmark$ b-jet sample-b-tagging procedure based on NN algorithm (secondary vertex, impact parameter...)

$\checkmark$ Light quark jet sample (decays $W \rightarrow q \bar{q}^{\prime}$ )

Jet purities

- $\boldsymbol{l}+$ jets: $\boldsymbol{b}$-jets $(88.5 \pm 5.7) \%$, light jets $(66.2 \pm 4.1) \%$

- Dilepton: $\boldsymbol{b}$-jets $\left(99.3^{+0.7}{ }_{-6.5}\right) \%$
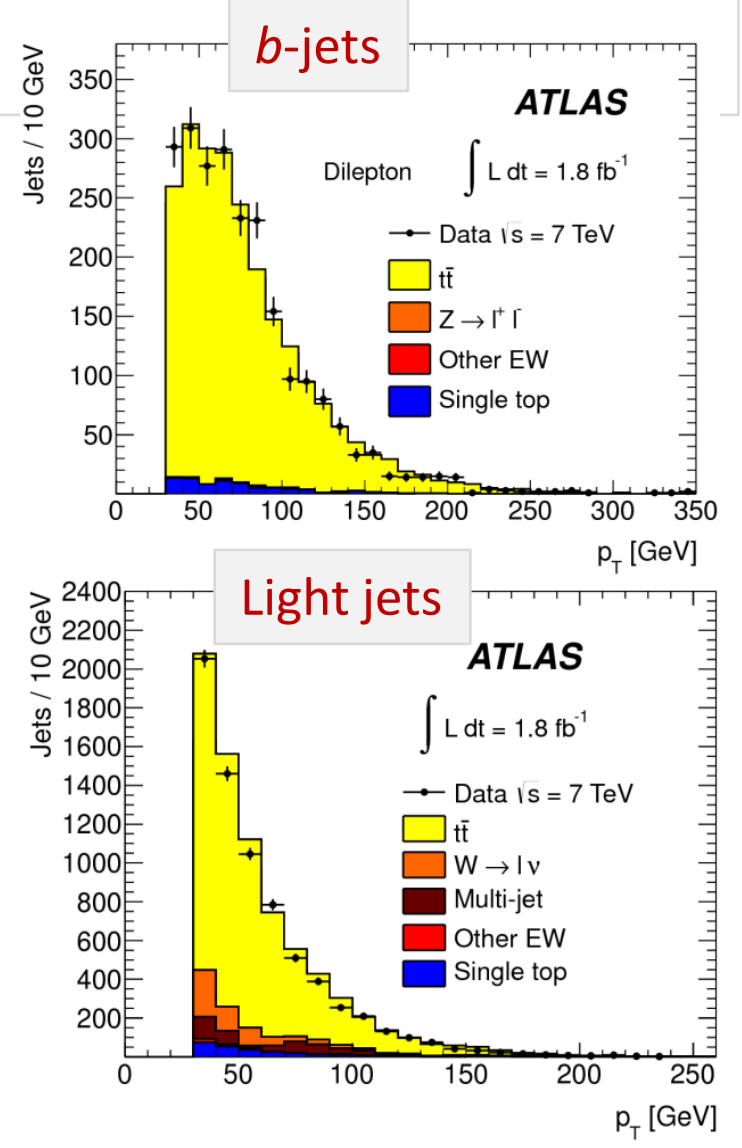


\section{Jet shapes in tt-bar events in $p p$ at $7 \mathrm{TeV}$}

Differential jet shape

$$
\rho(r)=\frac{1}{\Delta r} \frac{p_{T}(r-\Delta r / 2, r+\Delta r / 2)}{p_{T}(0, R)}
$$

\section{Integrated jet shape}

$$
\Psi(r)=\frac{p_{T}(0, r)}{p_{T}(0, R)}, r<R
$$

$\mathrm{r} \equiv$ distance to the jet axis in the $\eta-\varphi$ plane and $\mathrm{p}_{\mathrm{T}}\left(\mathrm{r}_{1}, \mathrm{r}_{2}\right) \equiv$ scalar sum of the $p_{\mathrm{T}}$ of the jet constituents with radii between $r_{1}$ and $r_{2}$.
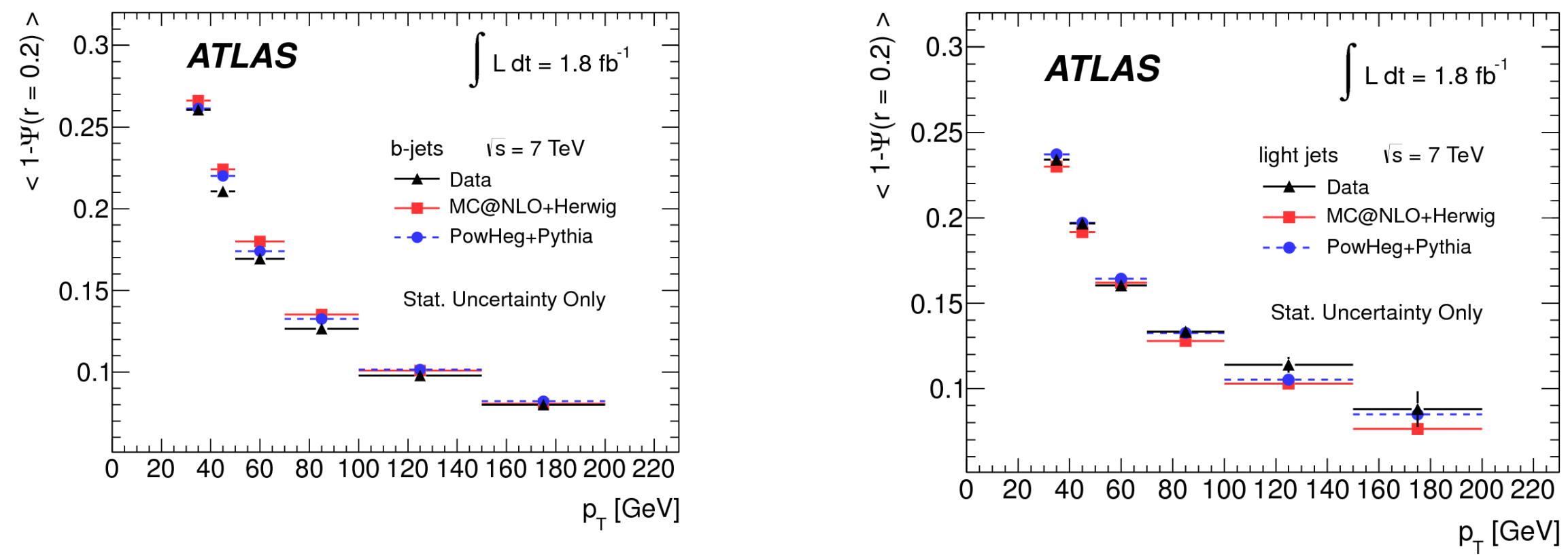

Dependence of the $b$-jet (left) and light-jet (right) shapes on the jet $p_{\mathrm{T}}$. $<1-\psi(r=0.2)>\equiv$ fraction of energy in the outer part of jet cone (l+jets events). 


\section{Jet shapes and uncertainties}
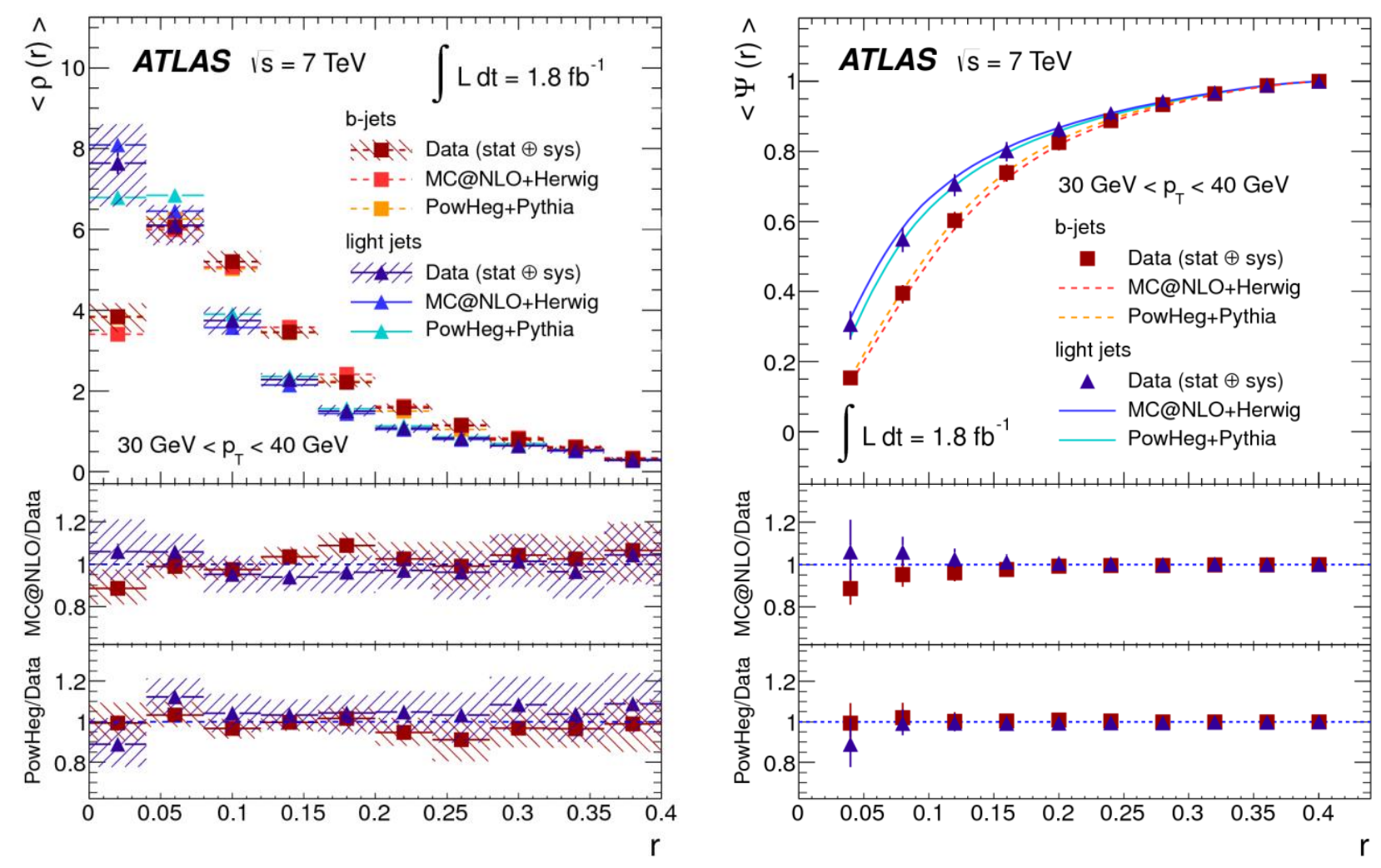

Data vs theory:

good agreement (see also p. 24)

Diff. shape systematics:

$\checkmark$ energy of individual clusters inside jet is varied : $2-10 \%$.

$\checkmark$ et energy resolution: $5 \%$.

$\checkmark$ Pile-up (vs r): $2-10 \%$.

$\checkmark$ Unfolding (3 MC generators used): $1-8 \%$.

Differential (integrated) jet shapes $\langle\rho(r)\rangle(\langle\Psi(r)>)$ vs $\boldsymbol{r}$ for light jets (triangles) and $\boldsymbol{b}$-jets (squares). The data vs different $\mathrm{MC}$ for $30 \mathrm{GeV}<\boldsymbol{p}_{\mathrm{T}}<40 \mathrm{GeV}$.

The uncertainties: statistical $\oplus$ systematic sources. 


\section{Conclusions}

$\square$ Jet physics provide us with powerful means for testing QCD - a lot of interesting results at $7 \mathrm{TeV}$ obtained.

$\square$ Measured differential cross section for

- Dijet production vs dijet mass and rapidity.

- Inclusive jet production $v s$ jet $\boldsymbol{p}_{T}$ and $\mathbf{y}$.

- Three-jet production vs $\mathrm{m}_{\mathrm{jjj}}$ and $\left|\mathrm{Y}^{*}\right|$.

Good consistency between the data and SM predictions found.

$\square$ Using di-jet events the quark composite scale $\Lambda$, excluded below $7 \mathrm{TeV}$.

$\square$ Flavor dijet decomposition for different jet combinations measured - giving good agreement with the SM predictions.

$\square$ Using $t \bar{t}$ events the $b$-jet and light jet shapes $v s$ distance to jet axis and jet $\boldsymbol{p}_{T}$ were measured - good agreement with SM expectations. 
Back up 


\section{Exclusion of contact interactions}

$\checkmark$ If quarks are composed of more fundamental particles with new strong interactions at a composite scale $\Lambda$ ( $\left.>\mathrm{m}_{\text {quark }}\right) \Rightarrow$ at energies $<<\Lambda$ : contact interaction from the underlying strong dynamics $\rightarrow$ observable effect.

$\checkmark$ Model of QCD + contact interactions (Cls) with left-left coupling and destructive interference between $\mathrm{Cls}$ and $\mathrm{QCD}$ is considered.

$\checkmark$ Measurement is restricted to the high dijet-mass $m_{12}>1.31 \mathrm{TeV}$ in the range $\quad y^{*}<0.5\left(\right.$ Cls: events preferentially produced at smaller $\left.y^{*}\right)$.

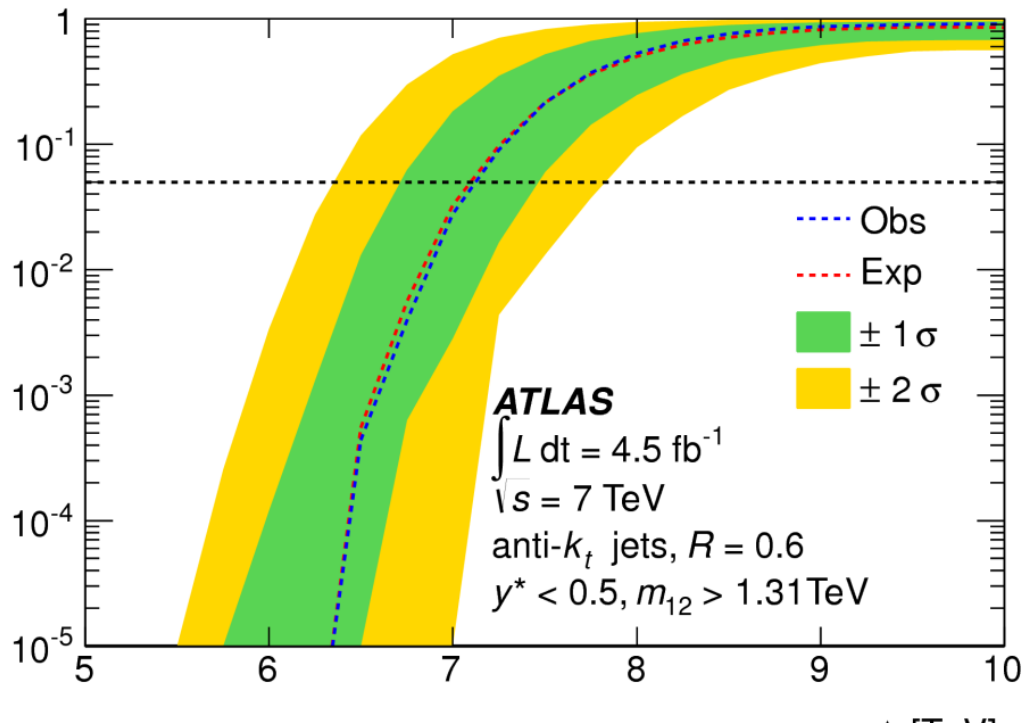

\begin{tabular}{|l|c|c|}
\hline \multicolumn{2}{|l|}{ PDF set } & \multicolumn{2}{|c|}{$\mathrm{R}=0.4$} \\
\hline \multicolumn{1}{|c|}{$\boldsymbol{\Lambda}[\mathrm{TeV}]$} & \multicolumn{1}{|c|}{ Exp } & Obs \\
\hline CT10 & 7.3 & 7.2 \\
\hline HERAPDF1.5 & 7.5 & 7.7 \\
\hline MSTW 2008 & 7.3 & 7.0 \\
\hline NNPDF2.1 & 7.3 & 7.2 \\
\hline
\end{tabular}

$\Lambda[\mathrm{TeV}]$

Measured cross section vs NLO QCD + Cls predictions: scan of CLs value for NLO QCD + Cls as a function of $\Lambda$, using the CT10 PDF set.... 


\section{Dijet flavour decomposition}

Flavour decomposition measurement: $\mathrm{pp}$ collision at $\sqrt{ }_{s}=7 \mathrm{TeV}, L_{\mathrm{int}}=39 \mathrm{pb}^{-1}$.

$\checkmark$ 3-level single jet trigger: events with jet $\boldsymbol{p}_{\mathrm{T}}$ over a threshold for $|\eta|<3.2$.

$\checkmark$ At least one reconstructed primary vertex candidate.

$\checkmark$ A candidate vertex: at least 10 tracks with $p_{\mathrm{T}}>150 \mathrm{MeV}$ associated to it.

$\checkmark$ Jets: used anti- $k_{t}$ algorithm with $R=0.4$, jets with $p_{\mathrm{T}}>30 \mathrm{GeV}+|\mathrm{y}|<2.1$ and 2 highest $p_{\mathrm{T}}$ jets with back-to-back topology: $\Delta \varphi>2.1$.

$\checkmark$ Investigated jet $p_{\mathrm{T}}$ intervals:

\begin{tabular}{|l|l|l|l|l|l|l|}
\hline Lead. jet $p_{\mathrm{T}}[\mathrm{GeV}]$ & $40-60$ & $60-80$ & $80-120$ & $120-160$ & $160-250$ & $250-500$ \\
\hline sLead. Jet $\mathrm{p}_{\mathrm{T}}[\mathrm{GeV}]$ & $30-60$ & $40-80$ & $50-120$ & $75-160$ & $100-250$ & $140-500$ \\
\hline
\end{tabular}

MC generators:

- Pythia 6.423 (LO parton matrix element for $2 \rightarrow 2$ processes) for template construction.

- NLO generator Powheg for interpretation of the analysis results. 


\section{3-jet production cross-section at $p p 7 \mathrm{TeV}$}

3-jet double diff. $x$-sec vs $m_{j j j}$ in 5 bins of $\left|Y^{*}\right|$ for jets with $R=0.6$ compared with NLOJET++ prediction with CT 10 PDF corrected for non-perturb. effects
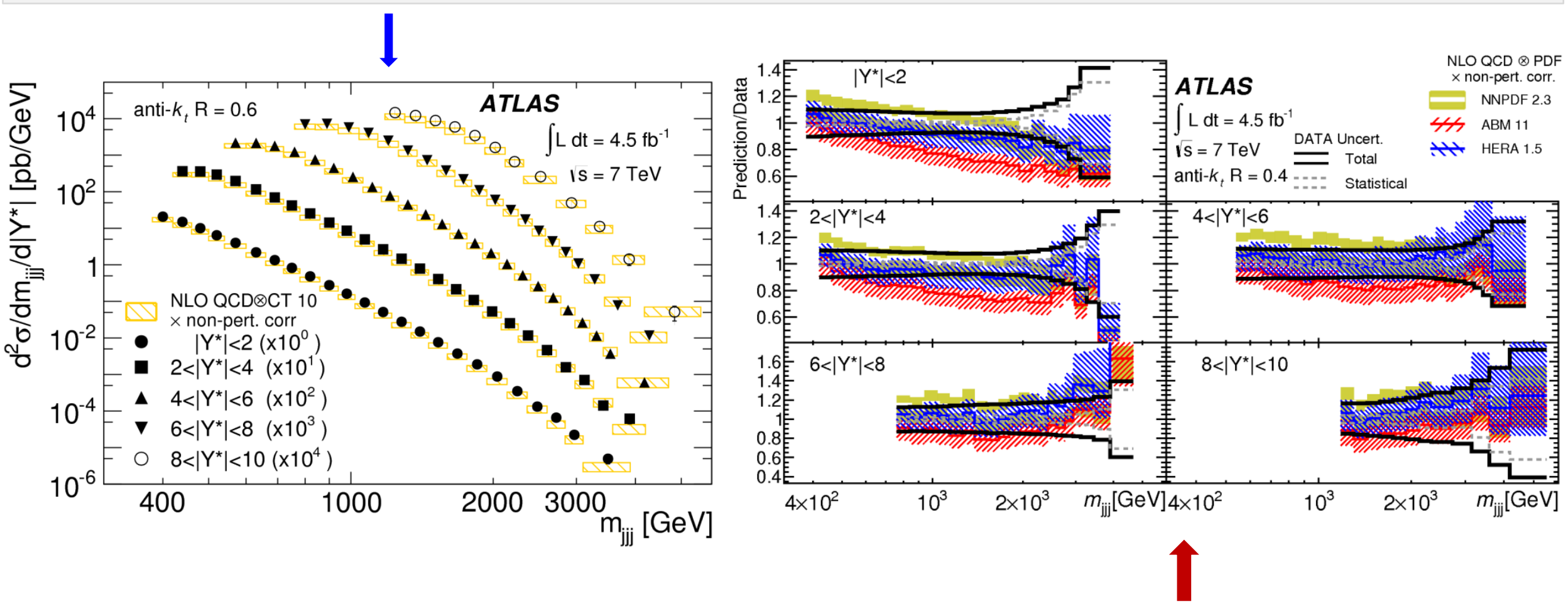

The ratio of NLO QCD predictions, obtained by using NLOJET++ with different PDF sets (NNPDF 2.3, ABM 11, HERA 1.5) and corrected for non-perturbative effects, to data as a function of $m_{j j j}$ in 5 bins of $\left|Y^{*}\right|$. 


\section{Diff. and integral jet shapes for jet $p_{T}$ intervals}
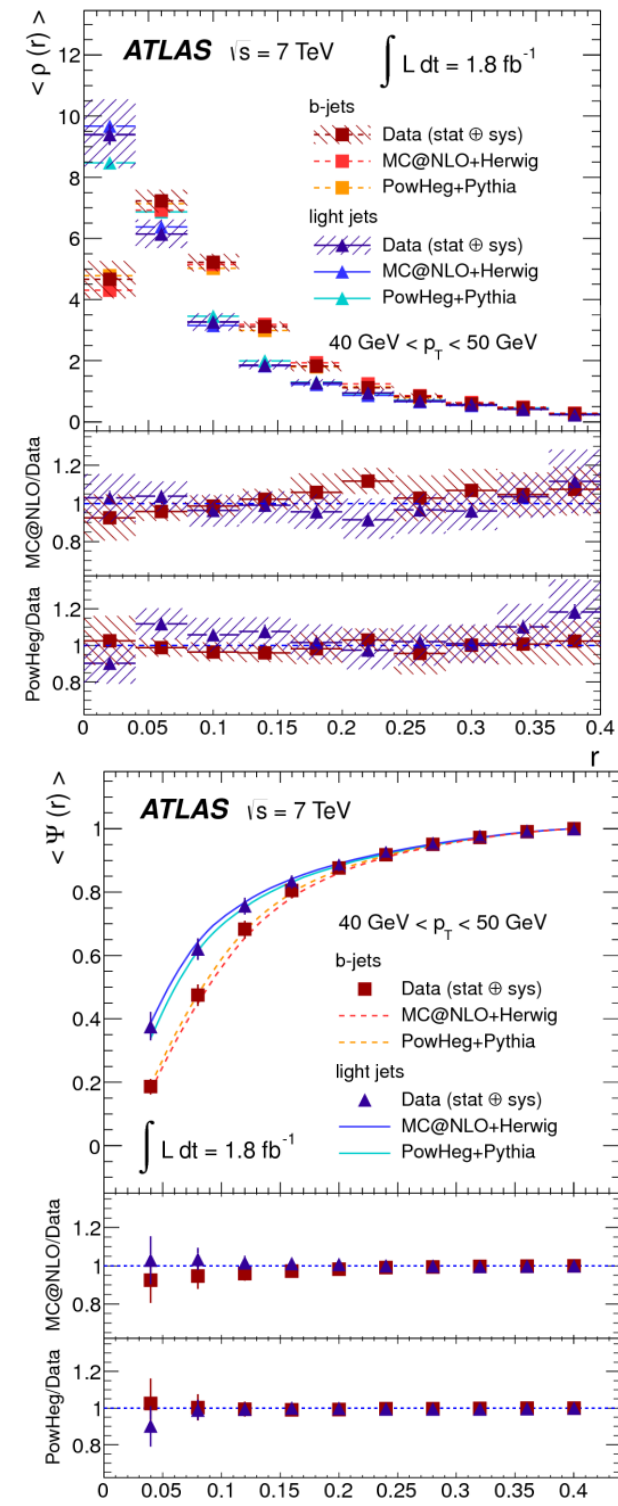

$7 / 2 / 2015$
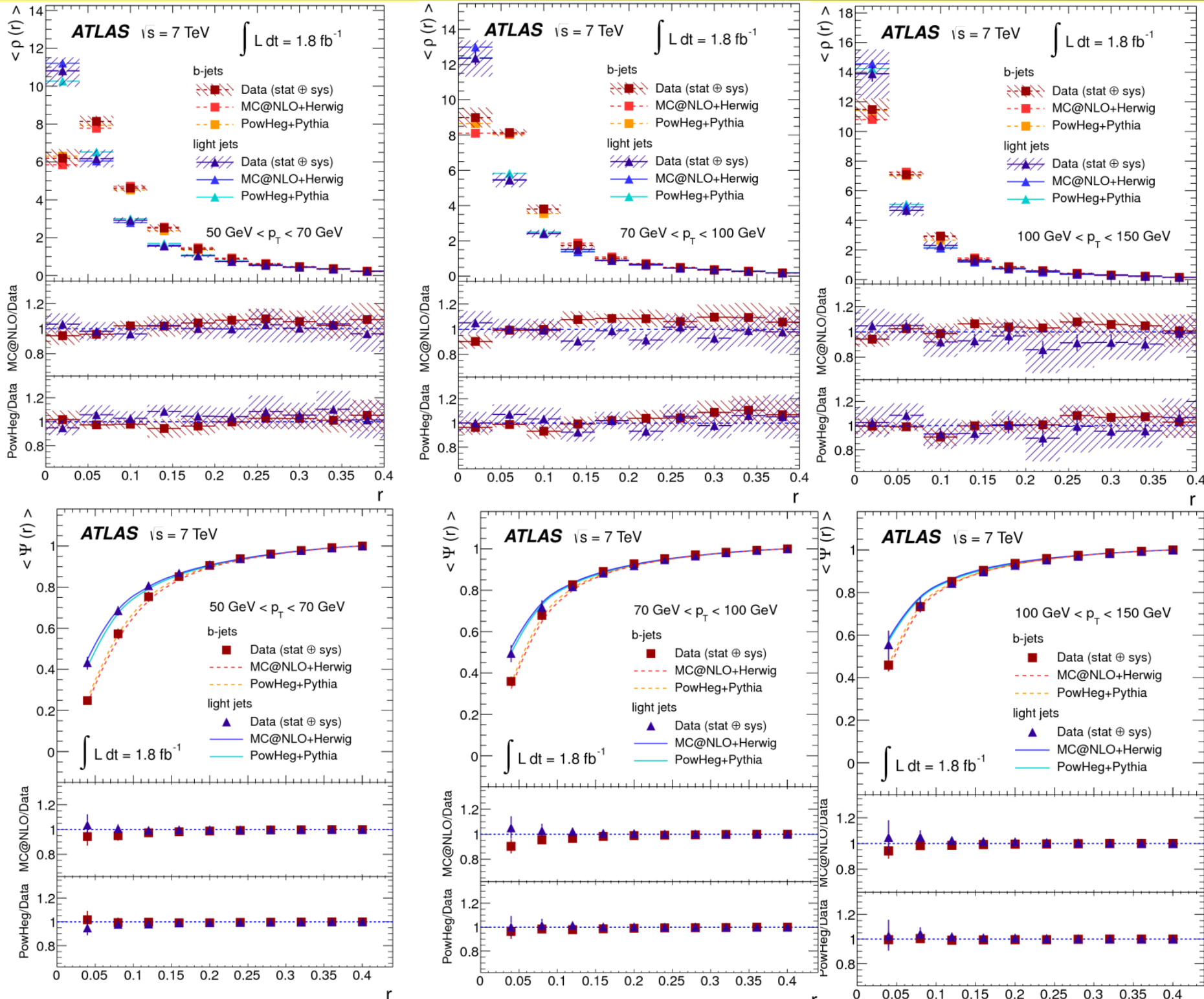

EDS2015, S. Tokar, Comenius U.

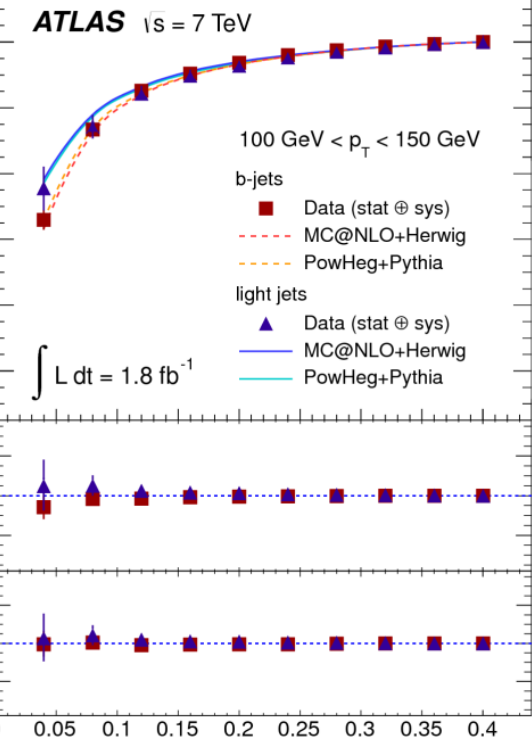

$\angle 0$ 